\title{
SG-0: A Small Standard Grid for DFT Quadrature on Large Systems
}

\author{
SIU-HUNG CHIEN, PETER M. W. GILL \\ Research School of Chemistry, Australian National University, Canberra, ACT 0200, Australia
}

Received 17 October 2005; Accepted 25 November 2005

DOI 10.1002/jcc.20383

Published online in Wiley InterScience (www.interscience.wiley.com).

\begin{abstract}
We report the development of a new standard quadrature grid for DFT calculations. Standard Grid 0 (SG-0) is designed to be approximately half as large as, and to provide approximately half the accuracy of, the established SG-1 grid. It is based on MultiExp and Lebedev quadrature for radial and angular coordinates, respectively. We find that SG-0 is typically 50\% faster than SG-1 for energy, gradient, and hessian calculations for the exchange-correlation energy. This leads to a $35-38 \%$ speedup in the total gradient and hessian computations, and we particularly recommend its use for preliminary calculations on moderately large biochemical systems. It has been implemented as the default grid for DFT calculations in the Q-Chem 3.0 package.
\end{abstract}

(C) 2006 Wiley Periodicals, Inc. J Comput Chem 27: 730-739, 2006

Key words: DFT; quadrature; grid error; numerical integration; standard quadrature grid

\section{Introduction}

The density functional theory (DFT) enjoys a number of significant advantages over traditional wave function-based post-Hartree-Fock quantum chemical methods: it is conceptually simpler; it is more easily implemented within software packages; and it is much less computationally costly. Nonetheless, notwithstanding the optimistic promises of the Hohenberg-Kohn theorem, ${ }^{1}$ it also possesses, at least in its present incarnations, several well-documented and fundamental deficiencies. First and foremost, it does not present a well-defined hierarchy of progressively more accurate approximations that lead, in principle, to the exact solution of the Schrödinger equation. ${ }^{2}$ Second, most popular versions include an improper energy contribution from the self-repulsion of electrons, leading to the insidious "self-interaction error" that plagues the application of DFT to systems with stretched or dissociating bonds. ${ }^{3}$ Third, its energy expression contains a term (the exchange-correlation contribution) which involves an integral that normally cannot be evaluated in closed form and must be estimated by an approximate quadrature. The canonical example of this third weakness is the Dirac-Slater exchange energy, which is proportional to the integral over all space of the $4 / 3$ power of the electron density. ${ }^{4}$

There have been a number of attempts to avoid the quadrature problem,,$^{5-8}$ and the best of these proceed by expanding the problematic integral in an auxiliary basis set. However, this tactic is less progressive than it appears because, in the final analysis, it only replaces the task of choosing optimal grid points with the task of selecting optimal auxiliary basis functions. Indeed, it is not difficult to show that such an expansion is mathematically equivalent to a quadrature, albeit in a different space. Moreover, it is found that one needs large auxiliary bases, increasing the computational expense and reducing the allure of such approaches. As a result, the overwhelming majority of contemporary DFT calculations continue to depend upon explicit quadrature.

Other groups, resigned to the inevitability of numerical integration but seeking to work optimally within that framework, have sought highly efficient quadrature methods, that is, ones that obtain high accuracy from relatively few grid points. ${ }^{9-18}$ Most of these can be classified as either "standard" models in which the grid points and weights are known beforehand, ${ }^{9-16}$ or "adaptive" schemes in which points and weights are chosen dynamically as the integrand is explored. ${ }^{17,18}$

More than a decade ago, we introduced SG-1, a standard grid that aimed to yield moderately accurate results at a low computational cost. ${ }^{11}$ It was designed to produce exchange-correlation energies with a computational effort comparable to that required to calculate the Coulomb energy using the linear-scaling Continuous Fast Multipole Method (CFMM) ${ }^{19}$ and includes approximately 3700 grid points per atom. Since that time, SG-1 has been widely used and is now established as a useful tool for preliminary explorations of potential energy surfaces. However, subsequent advances in the treatment of the Coulomb energy, principally the Fourier Transform Coulomb (FTC) method of Fusti-Molnar and

Correspondence to: S.-H. Chien; e-mail: chiensh@alumni.cuhk.net 
coworkers, ${ }^{20}$ have again shifted the balance, and there is now a need for a standard grid of approximately half the size of SG-1.

Increasing computational interest in large biochemical systems also necessitates the development of efficient and well-documented standard quadratures and, although more aggressively pruned grids are now available in some quantum chemical packages, their precise definitions and error analyses have not been published. To fill this gap, we have developed a new grid (SG-0) whose construction and validation are described here.

\section{Radial and Angular Quadratures}

Because of the presence of cusps in the electron density at each of a molecule's nuclei, ${ }^{21}$ quadrature in Cartesian coordinates is generally not recommended for computing molecular integrals. Instead, it is preferable to adopt the Becke partitioning scheme, ${ }^{9}$ wherein a molecular integral is reduced to a sum of atomic integrals, each of which is evaluated by quadrature in spherical polar coordinates. To approximate such an integral

$$
I=\int^{\infty} F(\mathbf{r}) d \mathbf{r}=\iint_{0}^{\pi 2 \pi} \int_{0} F(r, \vartheta, \varphi) r^{2} \sin \vartheta d \varphi d \varphi d r
$$

it is common to employ a product of radial and angular quadratures, viz:

$$
I \approx \sum_{i=1}^{N^{r}} w_{i}^{r} \sum_{j=1}^{N^{\Omega}} w_{j}^{\Omega} F\left(r_{i}, \vartheta_{j}, \varphi_{j}\right)
$$

in which $N^{\mathrm{r}}$ and $N^{\Omega}$ are the numbers of radial and angular nodes, respectively, and $w_{i}^{\mathrm{r}}$ and $w^{\Omega}{ }_{j}$ are the radial and angular weights, respectively.

Although Gauss-Laguerre and Gauss-Hermite quadratures are obvious choices for the radial integration over $[0, \infty)$, Treutler and Ahlrichs $\operatorname{argue}^{12}$ that these are nonoptimal because "the shell structures of atoms implies contributions of exponentials with a variety of orbital exponents." Instead, a number of schemes have been proposed to map the radial interval onto a finite one, usually [0,1], and then apply an appropriate quadrature. In 1993, Gill et al. chose to base SG-1 on the Euler-Maclaurin radial grid, ${ }^{10}$ but it is has been argued subsequently that the latter is not as effective as some other grids, including those of Becke, ${ }^{9}$ Treutler and Ahlrichs, ${ }^{12}$ and Mura and Knowles. ${ }^{13}$ In 2003, we introduced a new radial quadrature ${ }^{15}$ that combines the logarithmic transformation

$$
\begin{gathered}
r=-R \ln x \\
I=R^{3} \int_{0}^{1} x^{-1} f(r)\left(\ln ^{2} x\right) d x
\end{gathered}
$$

with the "log-squared" quadrature

$$
\int_{0}^{1} g(x)\left(\ln ^{2} x\right) d x \approx \sum_{i=1}^{n} a_{i} g\left(x_{i}\right)
$$

to yield the multi-exponential (MultiExp) grid

$$
\begin{gathered}
r_{i}=-R \ln x_{i} \\
w_{i}=-\left(\frac{a_{i}}{x_{i}}\right) R^{3} .
\end{gathered}
$$

By construction, MultiExp integrates certain linear combinations of exponentials exactly and, for this reason, appears particularly well suited to atomic quadrature. Its performance in evaluating exchange-correlation integrals in DFT calculations was studied by $\mathrm{us}^{15}$ and in a recent review by El-Sherbiny and Poirier. ${ }^{16}$

For the angular subintegration, separate $\vartheta$ and $\phi$ quadratures are possible but are generally less efficient ${ }^{22}$ than direct quadrature on the surface of a sphere ${ }^{23-25}$ and Koch and Holthausen ${ }^{26}$ have stated that "there seems to be a certain consensus that the so-called Lebedev grids offer the best value for money." A Lebedev grid of degree $l$ exactly integrates all spherical harmonics of degree $l$ or less and, in this sense, is a two-dimensional analog of the more familiar Gauss-Legendre grid in one dimension. Lebedev ${ }^{23}$ originally published grids up to $l=53$ and, later, Delley ${ }^{27}$ published grids up to $l=59$. More recently, Lebedev and Laikov have extended this work as far as the $l=131$ grid, which consists of 5810 points. $^{25}$ Fortunately, such high-order quadratures are not necessary for our purposes and SG-0 utilizes only the 170-point $(l=21)$ grid and its smaller cousins. For benchmarking, we have used the 1202-point $(l=59)$ grid.

\section{Benchmarks and Grid Optimization}

In this section, we discuss the platforms, programs, and theoretical levels used in this work, the training molecule sets and benchmarks, the scale factors used in the MultiExp grid and, finally, the procedure for the construction of SG-0.

\section{Hardware, Software and Theoretical Level}

Molecular energies were calculated at the B-LYP/6-31G(d) level using geometries optimized at the MP2/6-31G(d) level. The structural optimizations and vibrational frequency calculations discussed later were also performed at the B-LYP/6-31G(d) level. All quantum chemical calculations in this work were carried out by a modified version of the Q-Chem 2.1 package $^{28}$ on various platforms, including a 152-node linux-PC cluster, in which each node contains a $2.66 \mathrm{GHz}$ Intel-P4 processor, a Linux-PC workstation with dual 1.7-GHz Intel-P4 processors, and an Apple PowerMac workstation with dual 2.0-GHz G5 processors.

\section{Training Molecule Sets}

To ensure that our grid performs well in a variety of chemically important situations, our training set is biased toward compounds that are rich in the four most important elements, viz. $\mathrm{H}, \mathrm{C}, \mathrm{N}$, and 
O. The training set for carbon is the largest, comprising 113 species. Analogous sets for hydrogen, nitrogen, and oxygen contained 45, 33, and 35 species, respectively. For the other elements studied in this work, the training sets comprise up to 21 species. The training sets were designed to include each atom in a variety of chemical environments. For example, $\mathrm{NaF}, \mathrm{Na}_{2}$ and $\mathrm{KNa}$ are present in the training set for sodium, for these contain sodium atoms that are ionically positive, covalently neutral, and ionically negative, respectively. The molecules in a training set were selected from a pool consisting of the G2 and G3 molecules, ${ }^{29,30}$ as well as some simple inorganic molecules containing second-row elements. For brevity, lists of these species are not given here, but they can be obtained in electronic format from our Web page. ${ }^{31}$

\section{Benchmarks}

Martin et al. ${ }^{32}$ have proposed that the combination of the 99-point Euler-Maclaurin radial grid and the 974-point Lebedev angular grid, viz. EML $(99,974)$, yields results with minimal grid error but with an "unacceptably high premium in terms of CPU time" for routine calculations. In the present work, however, an even larger grid, EML(100,1202), was employed to obtain benchmark "exact" results.

During the optimization of the SG-0 grid for a given atom, the EML $(100,1202)$ grid was used on all other atoms. By comparing such calculations with those in which the $\operatorname{EML}(100,1202)$ grid was used on all atoms, we were able to calculate absolute atomization energy errors and the mean $\Delta E$ of these errors across a training set is used to measure the overall error associated with the SG-0 grid for that atom.

\section{Radial Scale Factors}

Each of the radial grids discussed above is completely defined by two parameters, the number of grid points $N^{\mathrm{r}}$ and the scale factor $R$, and the quadrature accuracy depends upon both of these. We have found that $R$ is particularly important in the MultiExp grid and we have optimized this carefully for each atom.

The mean absolute atomization energy error $\Delta E$ of an element can be calculated over a range of $R$ values and it is sometimes useful to graph these. Such profiles for hydrogen, carbon, nitrogen, and oxygen are shown in Figure 1 and reveal that $\Delta E$ oscillates when $R$ is too small or too large, but that the amplitudes of these oscillations diminish as $N$ increases. Such oscillations have been noted by other researchers, ${ }^{12,13}$ and Mura and Knowles argue that they arise for small $R$ because the resulting grids are not sufficiently diffuse to treat the outer regions of the electron density. ${ }^{13}$ Treutler and Ahlrichs suggest that the oscillations when $R$ is large arise from the close approach of the grid points to a neighboring nucleus. ${ }^{12}$ Mura and Knowles also propose that the amplitudes of these oscillations can be used as an indicator of the inherent numerical inaccuracy of the radial grids. It therefore follows that an optimal radial grid will have an $R$ value within the range where both the frequency and the amplitudes are small. We adopted this idea to obtain optimal $R$ values for SG-0.

Figure 1 shows that the frequencies and amplitudes of the $\Delta E$ values for the 20-, 23-, 25-, and 27-point grids decrease rapidly in the range of $1.1<R<1.6$ for carbon and, on this basis, the 23-point grid with $R=1.2$ was adopted for this element. Other elements were examined in the same way and it was found that a 23-point grid was satisfactory for $\mathrm{H}$ to $\mathrm{F}$ but that a 26-point grid was required for $\mathrm{Na}$ to $\mathrm{Cl}$. The associated $R$ values are given in Table 1 .

Our "parent grids" were obtained by combining these radial grids with the 170-point Lebedev angular grid. These can be abbreviated as $\operatorname{ML}(23,170)$ for first-row atoms and $\operatorname{ML}(26,170)$ for the second row elements.

\section{Grid Optimization}

As discussed above, our target was that SG-0 should be roughly half as large as SG-1 (i.e., about 1500 grid points per atom) but somewhat less accurate than SG-1 (i.e., yielding roughly double the SG-1 grid error). However, when we optimized the grid to achieve the first criterion, it was not always possible to satisfy the second.

In SG-1, an atom is partitioned into five radial zones and different angular grid is applied within each of these. This zoning approach was not satisfactory, however, for SG-0 because the MultiExp radial nodes are distributed very differently from those in the Euler-Maclaurin scheme. ${ }^{15}$ Finally, we decided to select the angular grid independently for each radial node. To optimize the SG-0 grid for an element, we therefore began with the parent grid and then progressively pruned the angular grid at each radial point, while monitoring $\Delta E$ to ensure that its change was acceptably small. Proceeding in this way, we found that we could prune the innermost and outermost angular grids aggressively but that relatively modest pruning (if any) was possible for radial points in the valence region of the atom. The final result was a grid that was qualitatively similar to SG-1 but in which the pruning process was more highly resolved.

\section{Standard Grid 0}

In this section, we first give precise definitions of the SG-0 grids obtained above. We then assess the accuracy of the new quadrature for predicting atomization energies, structures, and vibrational frequencies. Finally, we assess the computational time saving by this new scheme.

\section{Definition}

The SG-0 grid for each of the first- and second-row elements studied is given in Table 1. Each grid is defined by the number $N^{r}$ of radial grid points, the radial scale factor $R$, and the number of angular grid points at each of the radial points. The total number $N_{\text {tot }}$ of grid points on each atom is also listed and compared with the analogous value for the SG- 1 grid. The SG-0 grid for any element not listed in Table 1 is defined to be the same as the SG-1 grid for that element.

In the second column of Table 1 , the notation $x^{y}$ indicates that the $x$-point Lebedev grid is used at $y$ successive radial points. For example, the SG-0 grid for the hydrogen atom has 23 radial points and the configuration $6^{6} 18^{3} 26^{1} 38^{1} 74^{1} 110^{1} 146^{1} 86^{1} 50^{1} 38^{1} 18^{1}$ indicates that a six-point Lebedev angular grid is used at each of the six innermost radial points, an 18-point angular grid at each of the next three radial points, followed by a single 26-point grid, a single 38-point grid, and so forth. 

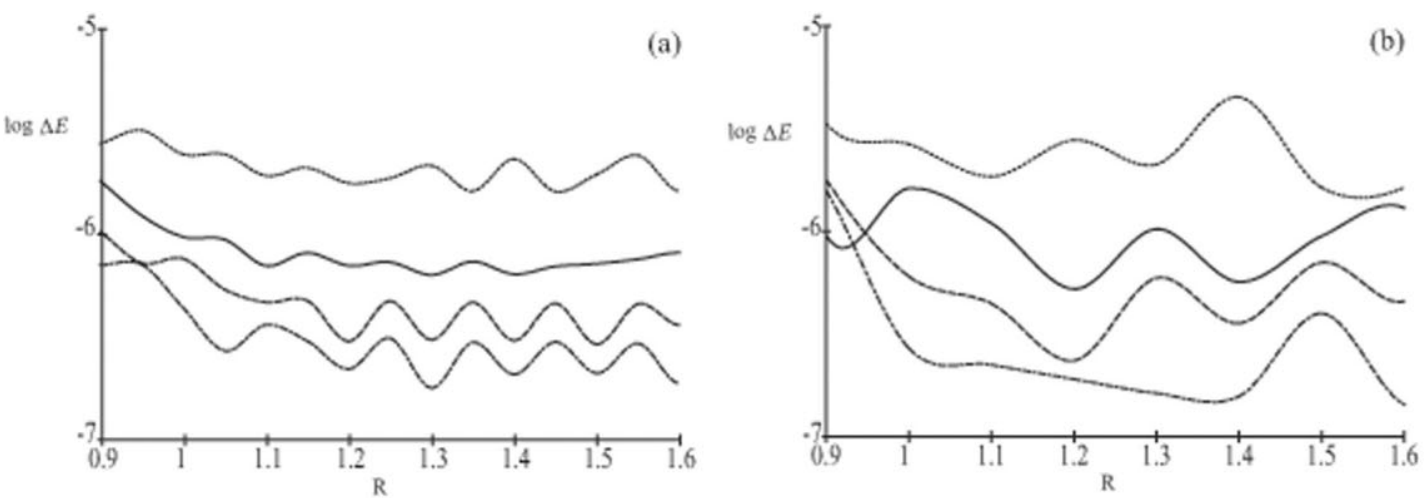

(b)
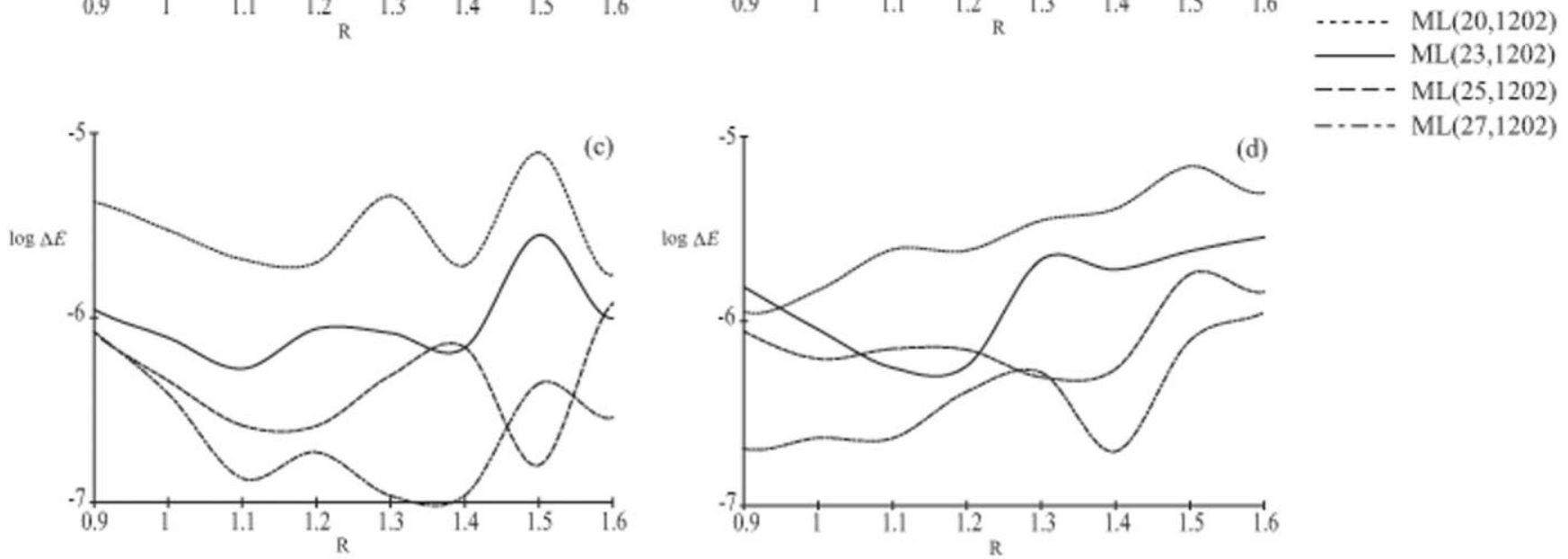

Figure 1. Variation of $\Delta E$ with $R$ for (a) hydrogen, (b) carbon, (c) nitrogen, and (d) oxygen.

Table 1 indicates that the SG-1 grid utilizes roughly 3800 points per atom and that SG-0 is about $40 \%$ as large. However, because of various cutoff strategies employed within the Q-
Chem program, the effective size of the SG-1 is only about 2800 and the practical reduction in moving to SG-0 is diminished accordingly.

Table 1. Lebedev Partition, Number $N_{\text {tot }}$ of Radial Points and Scale Factor $R$ in the SG-0 Grid.

\begin{tabular}{|c|c|c|c|c|c|}
\hline \multirow[b]{2}{*}{ Element } & \multirow[b]{2}{*}{ Lebedev partition } & \multirow[b]{2}{*}{$N$} & \multirow[b]{2}{*}{$R$} & \multicolumn{2}{|c|}{$N_{\text {tot }}$} \\
\hline & & & & SG-0 & SG-1 \\
\hline $\mathrm{H}$ & $6^{6}, 18^{3}, 26^{1}, 38^{1}, 74^{1}, 110^{1}, 146^{6}, 86^{1}, 50^{1}, 38^{1}, 18^{1}$ & 23 & 1.30 & 1406 & 3752 \\
\hline $\mathrm{Li}$ & $6^{6}, 18^{3}, 26^{1}, 38^{1}, 74^{1}, 110^{1}, 146^{6}, 86^{1}, 50^{1}, 38^{1}, 18^{1}$ & 23 & 1.95 & 1406 & 3816 \\
\hline $\mathrm{Be}$ & $6^{4}, 18^{2}, 26^{1}, 38^{2}, 74^{1}, 86^{1}, 110^{2}, 146^{5}, 50^{1}, 38^{1}, 18^{1}, 6^{2}$ & 23 & 2.20 & 1390 & 3816 \\
\hline B & $6^{4}, 26^{4}, 38^{3}, 86^{3}, 146^{6}, 38^{1}, 6^{2}$ & 23 & 1.45 & 1426 & 3816 \\
\hline $\mathrm{C}$ & $6^{6}, 18^{2}, 26^{1}, 38^{2}, 50^{2}, 86^{1}, 110^{1}, 146^{1}, 170^{2}, 146^{2}, 86^{1}, 38^{1}, 18^{1}$ & 23 & 1.20 & 1390 & 3816 \\
\hline $\mathrm{N}$ & $6^{6}, 18^{3}, 26^{1}, 38^{2}, 74^{2}, 110^{1}, 170^{2}, 146^{3}, 86^{1}, 50^{2}$ & 23 & 1.10 & 1414 & 3816 \\
\hline $\mathrm{O}$ & $6^{5}, 18^{1}, 26^{2}, 38^{1}, 50^{4}, 86^{1}, 110^{5}, 86^{1}, 50^{1}, 38^{1}, 6^{1}$ & 23 & 1.10 & 1154 & 3816 \\
\hline $\mathrm{F}$ & $6^{4}, 38^{2}, 50^{4}, 74^{2}, 110^{2}, 146^{2}, 110^{2}, 86^{3}, 50^{1}, 6^{1}$ & 23 & 1.20 & 1494 & 3816 \\
\hline $\mathrm{Na}$ & $6^{6}, 18^{2}, 26^{3}, 38^{1}, 50^{2}, 110^{8}, 74^{2}, 6^{2}$ & 26 & 2.30 & 1328 & 3760 \\
\hline $\mathrm{Mg}$ & $6^{5}, 18^{2}, 26^{2}, 38^{2}, 50^{2}, 74^{1}, 110^{2}, 146^{4}, 110^{1}, 86^{1}, 38^{2}, 18^{1}, 6^{1}$ & 26 & 2.20 & 1492 & 3760 \\
\hline $\mathrm{Al}$ & $6^{6}, 18^{2}, 26^{1}, 38^{2}, 50^{2}, 74^{1}, 86^{1}, 146^{2}, 170^{2}, 110^{2}, 86^{1}, 74^{1}, 26^{1}, 18^{1}, 6^{1}$ & 26 & 2.10 & 1496 & 3760 \\
\hline $\mathrm{Si}$ & $6^{5}, 18^{4}, 38^{4}, 50^{3}, 74^{1}, 110^{2}, 146^{1}, 170^{3}, 86^{1}, 50^{1}, 6^{1}$ & 26 & 1.30 & 1496 & 3760 \\
\hline $\mathrm{P}$ & $6^{5}, 18^{4}, 38^{4}, 50^{3}, 74^{1}, 110^{2}, 146^{1}, 170^{3}, 86^{1}, 50^{1}, 6^{1}$ & 26 & 1.30 & 1496 & 3760 \\
\hline S & $6^{4}, 18^{1}, 26^{8}, 38^{2}, 50^{1}, 74^{2}, 110^{1}, 170^{3}, 146^{1}, 110^{1}, 50^{1}, 6^{1}$ & 26 & 1.10 & 1456 & 3760 \\
\hline $\mathrm{Cl}$ & $6^{4}, 18^{7}, 26^{2}, 38^{2}, 50^{1}, 74^{1}, 110^{2}, 170^{3}, 146^{1}, 110^{1}, 86^{1}, 6^{1}$ & 26 & 1.45 & 1480 & 3760 \\
\hline
\end{tabular}

The total number $N_{\text {tot }}$ of grid points in SG-0 and SG-1 are included for comparison. 
Table 2. Total Energies and Atomization Energies ${ }^{a}$ Calculated Using Various Grids ${ }^{b}$.

\begin{tabular}{|c|c|c|c|c|c|c|}
\hline \multirow[b]{2}{*}{ Molecule } & \multicolumn{3}{|c|}{ Total energy } & \multicolumn{3}{|c|}{ Atomization energy } \\
\hline & $\operatorname{EML}(100,1202)$ & SG-1 & SG-0 & $\operatorname{EML}(100,1202)$ & SG-1 & SG-0 \\
\hline $\mathrm{H}$ & -0.495446 & 0 & 0 & - & - & - \\
\hline $\mathrm{Li}$ & -7.480142 & 0 & -9 & - & - & - \\
\hline $\mathrm{Be}$ & -14.656321 & -1 & 45 & - & - & - \\
\hline B & -24.641275 & 5 & 167 & - & - & - \\
\hline $\mathrm{C}$ & -37.832018 & 1 & 187 & - & - & - \\
\hline $\mathrm{N}$ & -54.568401 & 0 & 216 & - & - & - \\
\hline $\mathrm{O}$ & -75.046960 & 14 & -33 & - & - & - \\
\hline $\mathrm{F}$ & -99.702142 & 14 & -1663 & - & - & - \\
\hline $\mathrm{Na}$ & -162.266114 & 0 & 4377 & - & - & - \\
\hline $\mathrm{Mg}$ & -200.065580 & -48 & 2338 & - & - & - \\
\hline $\mathrm{Al}$ & -242.353526 & 13 & -1636 & - & - & - \\
\hline $\mathrm{Si}$ & -289.355676 & 18 & 190 & - & - & - \\
\hline $\mathrm{P}$ & -341.239900 & 12 & 5235 & - & - & - \\
\hline S & -398.087008 & 15 & -523 & - & - & - \\
\hline $\mathrm{Cl}$ & -460.117577 & 11 & 7077 & - & - & - \\
\hline $\mathrm{LiH}$ & -8.066114 & -3 & 12 & 0.090525 & 3 & -21 \\
\hline $\mathrm{BeH}$ & -15.242333 & -5 & 38 & 0.090565 & 5 & 8 \\
\hline $\mathrm{CH}$ & -38.460522 & -5 & 204 & 0.133058 & 6 & -17 \\
\hline $\mathrm{CH}_{2}\left({ }^{3} B_{1}\right)$ & -39.122778 & -2 & 183 & 0.299868 & 3 & 5 \\
\hline $\mathrm{CH}_{2}\left({ }^{1} A_{1}\right)$ & -39.102989 & -7 & 163 & 0.280078 & 8 & 24 \\
\hline $\mathrm{CH}_{3}$ & -39.805124 & -2 & 167 & 0.486767 & 3 & 20 \\
\hline $\mathrm{CH}_{4}$ & -40.478844 & -52 & 166 & 0.665041 & 53 & 21 \\
\hline $\mathrm{NH}$ & -55.200913 & 0 & 214 & 0.137067 & -1 & 2 \\
\hline $\mathrm{NH}_{2}$ & -55.849260 & 7 & 220 & 0.289967 & -8 & -5 \\
\hline $\mathrm{NH}_{3}$ & -56.518219 & 9 & 166 & 0.463480 & -10 & 50 \\
\hline $\mathrm{OH}$ & -75.707277 & 10 & -71 & 0.164871 & 4 & 39 \\
\hline $\mathrm{H}_{2} \mathrm{O}$ & -76.388313 & 9 & 7 & 0.350460 & 5 & -40 \\
\hline $\mathrm{HF}$ & -100.404438 & 3 & -1595 & 0.206850 & 11 & -68 \\
\hline $\mathrm{SiH}_{2}\left({ }^{3} B_{1}\right)$ & -290.551688 & -7 & 138 & 0.205120 & 25 & 52 \\
\hline $\mathrm{SiH}_{2}\left({ }^{1} A_{1}\right)$ & -290.584774 & 70 & 188 & 0.238205 & -52 & 2 \\
\hline $\mathrm{SiH}_{3}$ & -291.195812 & 67 & 143 & 0.353798 & -50 & 47 \\
\hline $\mathrm{SiH}_{4}$ & -291.839765 & 152 & 401 & 0.502304 & -135 & -211 \\
\hline $\mathrm{PH}_{2}$ & -342.474988 & 97 & 5347 & 0.244195 & -85 & -112 \\
\hline $\mathrm{PH}_{3}$ & -343.104301 & 18 & 5202 & 0.378062 & -6 & 34 \\
\hline $\mathrm{H}_{2} \mathrm{~S}$ & -399.356293 & -48 & -652 & 0.278393 & 63 & 129 \\
\hline $\mathrm{HCl}$ & -460.771784 & -29 & 6972 & 0.158761 & 40 & 104 \\
\hline $\mathrm{Li}_{2}$ & -14.992518 & -1 & -28 & 0.032234 & 1 & 11 \\
\hline $\mathrm{LiF}$ & -107.400576 & 124 & -1747 & 0.218292 & -110 & 76 \\
\hline $\mathrm{HCCH}$ & -77.291225 & -5 & 418 & 0.636297 & 7 & -43 \\
\hline $\mathrm{H}_{2} \mathrm{CCH}_{2}$ & -78.536820 & 13 & 517 & 0.890999 & -11 & -143 \\
\hline $\mathrm{H}_{3} \mathrm{CCH}_{3}$ & -79.762628 & 23 & 342 & 1.125915 & -21 & 33 \\
\hline $\mathrm{CN}\left({ }^{2} \Sigma_{\mathrm{g}}\right)$ & -92.695865 & -8 & 395 & 0.295446 & 8 & 8 \\
\hline $\mathrm{HCN}$ & -93.399585 & -7 & 428 & 0.503720 & 7 & -25 \\
\hline $\mathrm{CO}$ & -113.293977 & -10 & 213 & 0.414999 & 25 & -59 \\
\hline $\mathrm{HCO}$ & -113.830201 & -6 & 171 & 0.455776 & 21 & -16 \\
\hline $\mathrm{H}_{2} \mathrm{CO}$ & -114.471886 & -1 & 218 & 0.602016 & 16 & -63 \\
\hline $\mathrm{CH}_{3} \mathrm{OH}$ & -115.667550 & 15 & 205 & 0.806787 & 0 & -51 \\
\hline $\mathrm{N}_{2}$ & -109.510299 & -9 & 374 & 0.373498 & 7 & 57 \\
\hline $\mathrm{H}_{2} \mathrm{NNH}_{2}$ & -111.810636 & -1 & 373 & 0.692050 & -1 & 58 \\
\hline NO $\left({ }^{2} \mathrm{Z}\right)$ & -129.876874 & -4 & 168 & 0.261514 & 17 & 15 \\
\hline $\mathrm{O}_{2}$ & -150.315376 & -10 & -58 & 0.221456 & 38 & -7 \\
\hline $\mathrm{HOOH}$ & -151.512133 & -1 & -82 & 0.427320 & 28 & 17 \\
\hline $\mathrm{F}_{2}$ & -199.493140 & 33 & -3351 & 0.088856 & -4 & 25 \\
\hline $\mathrm{CO}_{2}$ & -188.562994 & -7 & 277 & 0.637056 & 36 & -155 \\
\hline $\mathrm{Na}_{2}$ & -324.560062 & -10 & 8848 & 0.027833 & 10 & -94 \\
\hline $\mathrm{Si}_{2}$ & -578.824880 & 46 & 322 & 0.113528 & -11 & 58 \\
\hline $\mathrm{P}_{2}$ & -682.663466 & 57 & 10498 & 0.183665 & -33 & -27 \\
\hline $\mathrm{S}_{2}$ & -796.335459 & 81 & -1252 & 0.161442 & -50 & 206 \\
\hline $\mathrm{Cl}_{2}$ & -920.317577 & 239 & 14075 & 0.082424 & -217 & 77 \\
\hline
\end{tabular}


Table 2. (Continued)

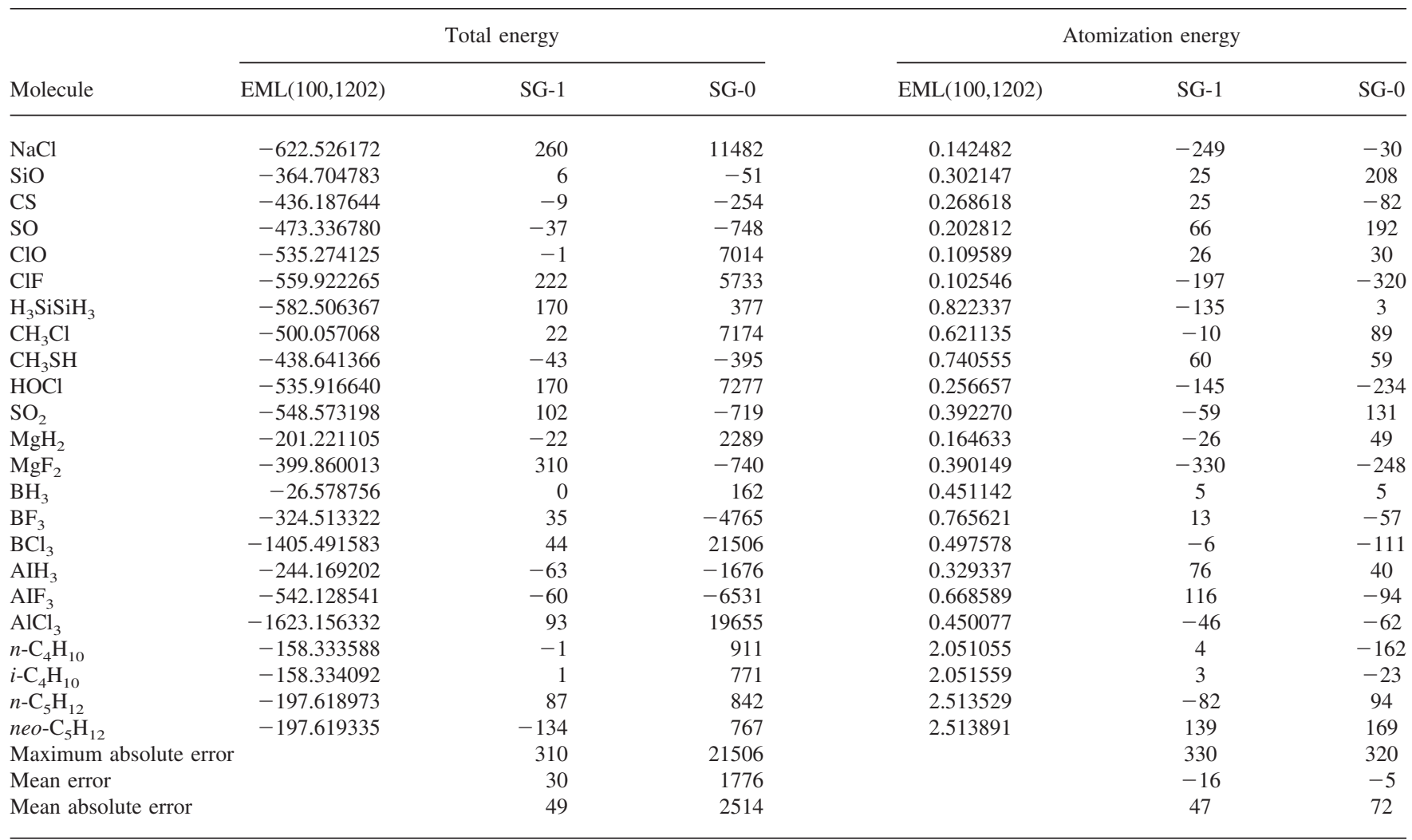

${ }^{a}$ Calculated at the B-LYP/6-31G(d) level, and based on the geometry optimized at the MP2/6-31G(d) level. Energies calculated using $\operatorname{EML}(100,1202)$ are in hartree; others are in microhartree relative to $\operatorname{EML}(100,1202)$.

${ }^{b} \operatorname{EML}\left(N^{r}, N^{\Omega}\right)$ is an Euler-Maclaurin-Lebedev grid with $N^{r}$ radial and $N^{\Omega}$ angular points.

It is interesting to note that, whereas most of the elements require at least one 146-point or 170-point angular grid, oxygen and sodium require nothing larger than the 110-point grid. The reason for this economy is not entirely clear to us but it suggests that, in molecular environments, the electron density of around these atoms tends to be more spherical than it is around other atoms.

\section{Accuracy}

\section{Atomization Energies}

Table 2 reports B-LYP/6-31G(d)//MP2/6-31G(d) total energies and atomization energies for 67 molecules, taken mainly from the G2 set. $^{28}$ In each case, the energies were computed using the large EML $(100,1202)$ grid, SG-1 and SG-0. By comparing these results and assuming that EML $(100,1202)$ gives negligible grid error, we can assess the SG-1 and SG-0 grid errors.

It is clear from Table 2 that the SG-0 total energies are very different from the SG-1 and EML $(100,1202)$ energies, but that the errors in SG-0 atomization energies are comparable to the corresponding SG-1 values. This suggests that most of the SG-0 grid error in total energies comes from the atomic core regions and that, to a large extent, these "core" errors cancel out in the calculation of atomization energies. The mean absolute deviation (MAD) of the SG-1 and SG-0 atomization energies are 47 and $72 \mu E_{h}$, respectively, indicating that the SG-0 error is typically less than twice the SG-1 error. The maximum absolute errors for SG-1 and SG-0 are 330 and $320 \mu E_{h}$, respectively.

Isomerization energies for $n$-butane $\rightarrow$ iso-butane and $n$-pentane $\rightarrow$ neo-pentane are good test cases for grid development. The energies (according to DFT) are only roughly $1 \mathrm{kcal} / \mathrm{mol}$ but, because the isomers' shapes are very different, the predicted energies are sensitive to grid deficiencies. Using the data in Table 2, the isomerization energies for the butane and pentane pairs are calculated to be 504 and $362 \mu E_{h}$, respectively. SG-1 overestimates these by 1 and $221 \mu E_{h}$, respectively, and SG-0 overestimates them by 139 and $75 \mu E_{h}$, respectively. The SG-0 errors are comparable with those of SG-1, suggesting that SG-0 should be useful for calculations of this type.

\section{Optimized Structural Parameters}

To assess the grid error in structure optimization, we have studied 45 small molecules with a total of 55 bond lengths, 19 bond angles, and 2 dihedral angles. These were optimized at the B-LYP/6-31G(d) level, and a selection of the resulting structural parameters are shown in Table 3. The full set is available as Supplementary Data. ${ }^{31}$

Compared with $\operatorname{EML}(100,1202)$ bond lengths, the maximum absolute deviation (MAX) in SG-1 is $0.00362 \AA(\mathrm{Mg}-\mathrm{F}$ bond 
Table 3. The Grid Errors Associated with SG-1 and SG-0 in Geometric Parameters That Optimized at the B-LYP/6-31G(d) Level.

\begin{tabular}{|c|c|c|c|c|}
\hline Molecule & Point Group ${ }^{a}$ & $\operatorname{EML}(100,1202)$ & $\mathrm{SG}-1^{b}$ & $\mathrm{SG}-0^{b}$ \\
\hline $\begin{array}{l}\mathrm{H}_{2} \\
r(\mathrm{HH})\end{array}$ & $D_{\infty h}$ & 0.74802 & -0.00002 & 0.00009 \\
\hline $\mathrm{Li}_{2}$ & $D_{\infty h}$ & & & \\
\hline$r(\mathrm{LiLi})$ & & 2.72808 & 0.00053 & 0.00148 \\
\hline $\mathrm{BeH}$ & $C_{\infty v}$ & & & \\
\hline$r(\mathrm{BeH})$ & & 1.35542 & -0.00020 & -0.00007 \\
\hline $\mathrm{BH}_{3}$ & $D_{3 h}$ & & & \\
\hline$r(\mathrm{BH})$ & & 1.19995 & -0.00001 & 0.00002 \\
\hline $\mathrm{H}_{3} \mathrm{CCH}_{3}$ & $D_{3 d}$ & & & \\
\hline$r(\mathrm{CC})$ & & 1.34100 & -0.00001 & -0.00098 \\
\hline$r(\mathrm{CH})$ & & 1.09483 & 0.00005 & 0.00009 \\
\hline$a(\mathrm{HCH})$ & & 116.23 & -0.01 & -0.12 \\
\hline $\mathrm{N}_{2}$ & $D_{\infty h}$ & & & \\
\hline$r(\mathrm{NN})$ & & 1.11812 & 0.00000 & -0.00011 \\
\hline $\mathrm{O}_{2}$ & $D_{\infty h}$ & & & \\
\hline$r(\mathrm{OO})$ & & 1.23949 & 0.00017 & 0.00036 \\
\hline $\mathrm{F}_{2}$ & $D_{\infty h}$ & & & \\
\hline$r(\mathrm{FF})$ & & 1.43370 & 0.00016 & -0.00091 \\
\hline $\mathrm{Na}_{2}$ & $D_{\infty h}$ & & & \\
\hline$r(\mathrm{NaNa})$ & & 3.04816 & 0.00134 & -0.01130 \\
\hline $\mathrm{MgF}_{2}$ & $D_{\infty h}$ & & & \\
\hline$r(\mathrm{MgF})$ & & 1.75086 & -0.00362 & -0.00387 \\
\hline $\mathrm{AlH}_{3}$ & $D_{3 h}$ & & & \\
\hline$r(\mathrm{AlH})$ & & 1.59797 & 0.00006 & -0.00064 \\
\hline $\mathrm{Si}_{2}$ & $D_{\infty h}$ & & & \\
\hline$r(\mathrm{SiSi})$ & & 2.32206 & 0.00104 & -0.00499 \\
\hline $\mathrm{P}_{2}$ & $D_{\infty h}$ & & & \\
\hline$r(\mathrm{PP})$ & & 1.92919 & 0.00040 & 0.00146 \\
\hline $\mathrm{S}_{2}$ & $D_{\infty h}$ & & & \\
\hline$r(\mathrm{SS})$ & & 1.95668 & -0.00004 & -0.00083 \\
\hline $\mathrm{Cl}_{2}$ & $D_{\infty h}$ & & & \\
\hline$r(\mathrm{ClCl})$ & & 2.07628 & 0.00268 & 0.00268 \\
\hline $\mathrm{SiH}_{2}\left({ }^{3} B_{1}\right)$ & $C_{2 v}$ & & & \\
\hline$r(\mathrm{SiH})$ & & 1.50309 & 0.00100 & 0.00004 \\
\hline$r(\mathrm{HSiH})$ & & 118.22 & -0.05 & -0.33 \\
\hline $\mathrm{H}_{3} \mathrm{COH}$ & $C_{s}$ & & & \\
\hline ( $\mathrm{H}_{a}$ in-plane, $\mathrm{H}_{b}$ out-of-plane) & & & & \\
\hline$r(\mathrm{CO})$ & & 1.43474 & 0.00274 & 0.00355 \\
\hline$r\left(\mathrm{CH}_{a}\right)$ & & 1.10098 & -0.00061 & -0.00090 \\
\hline$r\left(\mathrm{CH}_{b}\right)$ & & 1.10981 & -0.00068 & -0.00057 \\
\hline$r(\mathrm{OH})$ & & 0.98013 & 0.00020 & 0.00003 \\
\hline$a\left(\mathrm{OCH}_{a}\right)$ & & 106.40 & 0.01 & 0.11 \\
\hline$a(\mathrm{COH})$ & & 106.85 & -0.02 & -0.25 \\
\hline$a\left(\mathrm{H}_{b} \mathrm{CH}_{b}\right)$ & & 108.30 & -0.23 & -0.07 \\
\hline $\mathrm{HCO}$ & $C_{s}$ & & & \\
\hline$r(\mathrm{CO})$ & & 1.19578 & -0.00005 & -0.00001 \\
\hline$r(\mathrm{CH})$ & & 1.14080 & -0.00011 & -0.00036 \\
\hline$a(\mathrm{HCO})$ & & 122.92 & -0.01 & -0.11 \\
\hline $\mathrm{H}_{2} \mathrm{NNH}_{2}$ & $\mathrm{C}_{2}$ & & & \\
\hline$r(\mathrm{NN})$ & & 1.46237 & 0.00028 & -0.00088 \\
\hline$r\left(\mathrm{NH}_{b}\right)$ & & 1.03330 & -0.00001 & 0.00001 \\
\hline$r\left(\mathrm{NH}_{a}\right)$ & & 1.02765 & 0.00001 & 0.00011 \\
\hline$a\left(\mathrm{NNH}_{b}\right)$ & & 111.06 & 0.02 & 0.09 \\
\hline$a\left(\mathrm{NNH}_{\sigma}\right)$ & & 105.48 & 0.00 & 0.00 \\
\hline$a\left(\mathrm{H}_{a} N H_{\mathrm{b}}\right)$ & & 105.68 & -0.02 & 0.09 \\
\hline$d\left(\mathrm{H}_{a} \mathrm{NNH}_{b}\right)$ & & -90.39 & -0.26 & -0.51 \\
\hline $\mathrm{HOOH}$ & $C_{2}$ & & & \\
\hline$r(\mathrm{OO})$ & & 1.49390 & 0.00012 & -0.00034 \\
\hline$r(\mathrm{OH})$ & & 0.98554 & -0.00008 & 0.00017 \\
\hline$a(\mathrm{OOH})$ & & 98.47 & 0.00 & 0.11 \\
\hline$d(\mathrm{HOOH})$ & & 120.39 & 0.11 & -4.07 \\
\hline
\end{tabular}

Bond distance in angstroms, bond and dihedral angles in degrees.

${ }^{a}$ Symmetry constrains were turned off in the calculations at the B-LYP/6-31G(d) level.

${ }^{b}$ Quantities calculated by the SG-1 and SG-0 quadratures are given relative to $\operatorname{EML}(100,1202)$ values. 
Table 4. Frequencies (in $\mathrm{cm}^{-}$) Calculated at the B-LYP/6-31G(d) Level, Using the EML(100,1202), SG-1 and SG-0 Quadratures, and the Corresponding Zero Point Energy (ZPE, in $\mu$ hartree) ${ }^{a}$

\begin{tabular}{|c|c|c|c|c|}
\hline Molecule & & EML $(100,1202)$ & SG- $1^{b}$ & SG- $0^{b}$ \\
\hline \multirow[t]{2}{*}{$\mathrm{H}_{2}\left(D_{\alpha, h}\right)$} & $\Sigma_{g}$ & 4373.6 & 0.8 & -7.9 \\
\hline & ZPE & 9963 & 2 & -19 \\
\hline \multirow[t]{2}{*}{$\mathrm{Li}_{2}\left(D_{\alpha, h}\right)$} & $\Sigma_{g}$ & 331.7 & 1.1 & 0.9 \\
\hline & $\mathrm{ZPE}$ & 755 & 3 & 0 \\
\hline \multirow[t]{5}{*}{$\mathrm{BH}_{3}\left(D_{3 h}\right)$} & $A_{1}{ }^{\prime}$ & 2540.5 & -2.5 & -1.4 \\
\hline & $A_{2}^{\prime \prime}$ & 1141.2 & 1.2 & 0.8 \\
\hline & $E^{\prime}$ & 2669.7 & $-2.3(1)$ & $-1.1(1)$ \\
\hline & & 1185.5 & $0.6(1)$ & $0.7(8)$ \\
\hline & ZPE & 25954 & -11 & 8 \\
\hline \multirow[t]{6}{*}{$\mathrm{HCCH}\left(D_{\alpha, \mathrm{h} h}\right)$} & $\Sigma_{g}$ & 3459.1 & 0.1 & 1.5 \\
\hline & & 2020.9 & 0.2 & 0.2 \\
\hline & $\Sigma_{\mathrm{k}}$ & 3363.9 & 0.1 & 1.7 \\
\hline & $\Pi_{g}$ & 420.7 & 2.2 & -13.9 \\
\hline & $\Pi_{u}^{o}$ & 742.6 & 1.5 & -9.5 \\
\hline & $\mathrm{ZPE}$ & 25448 & 18 & -116 \\
\hline \multirow[t]{2}{*}{$\mathrm{N}_{2}\left(D_{\alpha, \mathrm{hh}}\right)$} & $\Sigma_{g}$ & 2336.4 & 3.5 & 3.6 \\
\hline & $\mathrm{ZPE}$ & 5323 & 8 & 0 \\
\hline \multirow[t]{2}{*}{$\mathrm{O}_{2}\left(D_{\alpha, \mathrm{h} h}\right)$} & $\Sigma_{\mathrm{g}}$ & 1516.6 & -0.8 & 2.5 \\
\hline & $\mathrm{ZPE}$ & 3455 & -2 & 8 \\
\hline \multirow[t]{2}{*}{$\mathrm{F}_{2}\left(D_{\alpha, \mathrm{hh}}\right)$} & $\Sigma_{g}$ & 988.0 & -5.3 & -6.5 \\
\hline & $\mathrm{ZPE}$ & 2250 & -11 & -3 \\
\hline \multirow[t]{2}{*}{$\mathrm{Na}_{2}\left(D_{\alpha, \mathrm{h} h}\right)$} & $\Sigma_{\mathrm{g}}$ & 153.8 & -12.9 & -0.1 \\
\hline & $\mathrm{ZPE}$ & 351 & -29 & 29 \\
\hline \multirow[t]{4}{*}{$\operatorname{MgF}_{2}\left(D_{\alpha, \mathrm{hh}}\right)$} & $\Sigma_{g}$ & 569.1 & 10.6 & 3.7 \\
\hline & $\Sigma_{u}$ & 898.7 & 13.8 & 2.2 \\
\hline & $\Pi_{u}$ & 134.4 & -18.8 & -28.3 \\
\hline & ZPE & 3957 & -30 & -86 \\
\hline \multirow[t]{5}{*}{$\mathrm{AlH}_{3}\left(D_{3 h}\right)$} & $A_{1}{ }^{\prime}$ & 1878.3 & 24.2 & 1.9 \\
\hline & $A_{2}^{\prime \prime}$ & 686.4 & 0.7 & 3.5 \\
\hline & $E^{\prime}$ & 1897.3 & $21.9(3)$ & $-1.0(1)$ \\
\hline & & 767.1 & -0.7 (1) & -1.5 (19) \\
\hline & ZPE & 17982 & 153 & -153 \\
\hline \multirow[t]{2}{*}{$\mathrm{Si}_{2}\left(D_{\alpha, \mathrm{h} h}\right)$} & $\Sigma_{g}$ & 453.9 & 5.6 & 9.9 \\
\hline & $\mathrm{ZPE}$ & 1034 & 13 & 10 \\
\hline \multirow[t]{2}{*}{$\mathrm{P}_{2}\left(D_{\alpha, \mathrm{h} h}\right)$} & $\Sigma_{g}$ & 750.4 & -7.5 & 13.4 \\
\hline & $\mathrm{ZPE}$ & 1710 & -18 & 48 \\
\hline \multirow[t]{2}{*}{$\mathrm{S}_{2}\left(D_{\alpha, \mathrm{h} h}\right)$} & $\Sigma_{g}$ & 652.1 & -11.8 & 10.4 \\
\hline & $\mathrm{ZPE}$ & 1485 & -27 & 51 \\
\hline \multirow[t]{2}{*}{$\mathrm{Cl}_{2}\left(D_{\alpha, \mathrm{h} h}\right)$} & $\Sigma_{g}$ & 481.8 & -10.2 & 11.6 \\
\hline & $\mathrm{ZPE}$ & 1098 & -24 & 49 \\
\hline \multirow[t]{4}{*}{$\mathrm{SiH}_{2}\left({ }^{1} A_{1}\right)\left(C_{2 v}\right)$} & $A_{1}$ & 1968.3 & 24.1 & 80.9 \\
\hline & & 1009.6 & -16.3 & -4.0 \\
\hline & $B_{2}$ & 1974.9 & 29.3 & 76.7 \\
\hline & $\mathrm{ZPE}$ & 11283 & 84 & 266 \\
\hline \multirow[t]{4}{*}{$\mathrm{PH}_{2}\left(C_{2 v}\right)$} & $A_{1}$ & 2284.1 & -65.0 & -80.4 \\
\hline & & 1119.8 & -19.9 & -27.2 \\
\hline & $B_{2}$ & 2298.6 & -65.3 & -71.1 \\
\hline & ZPE & 12991 & -343 & -64 \\
\hline \multirow[t]{4}{*}{$\mathrm{H}_{2} \mathrm{~S}\left(C_{2 v}\right)$} & $A_{1}$ & 2586.0 & 76.9 & 36.8 \\
\hline & & 1214.1 & 1.1 & 4.4 \\
\hline & $B_{2}$ & 2608.3 & 67.7 & 25.6 \\
\hline & ZPE & 14599 & 333 & -180 \\
\hline
\end{tabular}

${ }^{a}$ Quantities calculated using SG-1 and SG-0 are given relative to $\operatorname{EML}(100,1202)$ values.

${ }^{b}$ When the integration grid is small, degenerate modes in nonabelian molecules split slightly; comparison made in the table was based on their average values. Differences with magnitude larger than $1 \mathrm{~cm}^{-1}$ are given in parentheses. 
Table 5. CPU Time (in Seconds) for the Exchange-Correlation Energy Gradient and Hessian Calculations [B-LYP/6-31G(d)] for Amino Acids, as Well as the Total Number of Grid Points Used in SG-1 and SG-0.

\begin{tabular}{|c|c|c|c|c|c|c|}
\hline \multirow[b]{2}{*}{ Species } & \multicolumn{2}{|c|}{$N_{\text {tot }}$} & \multicolumn{2}{|c|}{ XC Gradient ${ }^{\mathrm{a}}$} & \multicolumn{2}{|c|}{ XC Hessian ${ }^{\mathrm{a}}$} \\
\hline & SG-1 & SG-0 & SG-1 & SG-0 & SG-1 & SG-0 \\
\hline Gly & 25338 & 12378 & $3(4)$ & $1(3)$ & 29 (172) & 14 (102) \\
\hline Ala & 33734 & 16580 & $5(7)$ & $3(5)$ & $65(450)$ & $32(270)$ \\
\hline Val & 50526 & 24984 & $14(19)$ & $7(12)$ & 218 (1835) & $110(1124)$ \\
\hline Leu & 58922 & 29186 & $18(25)$ & $9(16)$ & 339 (2909) & 172 (1794) \\
\hline Ile & 58922 & 29186 & $18(25)$ & $9(16)$ & 343 (2935) & 173 (1806) \\
\hline Ser & 36604 & 17734 & $7(11)$ & $4(7)$ & $92(716)$ & $46(438)$ \\
\hline Thr & 45000 & 21936 & $12(16)$ & $6(10)$ & 174 (1415) & 85 (846) \\
\hline Cys & 36462 & 18036 & $8(12)$ & $4(8)$ & $94(763)$ & 47 (479) \\
\hline Met & 53254 & 26440 & $15(23)$ & $7(15)$ & 240 (2095) & $121(1312)$ \\
\hline Asp & 42258 & 20278 & $12(18)$ & $6(12)$ & $162(1466)$ & 80 (906) \\
\hline Asn & 45064 & 21944 & $13(19)$ & $7(12)$ & 194 (1690) & 97 (1047) \\
\hline Glu & 50654 & 24480 & $16(23)$ & $8(15)$ & 237 (2170) & 117 (1339) \\
\hline Gln & 53460 & 26146 & $16(23)$ & $8(15)$ & $266(2392)$ & $132(1480)$ \\
\hline Arg & 70338 & 34834 & 27 (39) & $14(26)$ & $539(5181)$ & $275(3200)$ \\
\hline Lys & 64598 & 32006 & $19(27)$ & $10(17)$ & 378 (3225) & 193 (1983) \\
\hline His & 53438 & 26390 & $18(26)$ & $9(17)$ & $290(2855)$ & $148(1808)$ \\
\hline Phe & 61662 & 30544 & $25(36)$ & $13(24)$ & 434 (4478) & $221(2848)$ \\
\hline Tyr & 64532 & 31698 & $28(40)$ & $14(26)$ & $484(5197)$ & $249(3342)$ \\
\hline Trp & 72906 & 36144 & $37(54)$ & $19(36)$ & $685(8212)$ & $353(5341)$ \\
\hline Pro & 44914 & 22172 & $11(16)$ & $6(10)$ & $173(1411)$ & $86(863)$ \\
\hline \multicolumn{7}{|c|}{ Average percentage of grid-size reduction in SG-0 $=51 \%$} \\
\hline \multicolumn{7}{|c|}{ Average percentage CPU time saving: } \\
\hline \multicolumn{7}{|c|}{ XC Gradient $\mathrm{b}^{\mathrm{b}}=50 \%(35 \%)$} \\
\hline \multicolumn{7}{|c|}{$\mathrm{XC}$ Hessian $^{\mathrm{b}}=50 \%(38 \%)$} \\
\hline
\end{tabular}

${ }^{a}$ Total gradient and hessian times are given inside parentheses.

${ }^{\mathrm{b}}$ Time saving for total gradient and hessian are given inside parentheses.

length in $\mathrm{MgF}_{2}$ ) and the mean absolute deviation (MAD) of all the bond lengths is $0.00038 \AA$. For SG-0, the MAX is $0.01130 \AA$ $\left(\mathrm{Na}-\mathrm{Na}\right.$ distance in $\left.\mathrm{Na}_{2}\right)$ and the MAD is $0.00074 \AA$.

Compared with EML $(100,1202)$ bond angles, the MAX in SG-1 is $0.09^{\circ}\left(\angle \mathrm{H}_{\mathrm{b}}-\mathrm{C}-\mathrm{H}_{\mathrm{b}}\right.$ in $\left.\mathrm{H}_{3} \mathrm{COH}\right)$ and the MAD is $0.02^{\circ}$. For SG-0, the MAX is $0.33^{\circ}\left(\angle \mathrm{H}-\mathrm{Si}-\mathrm{H}\right.$ in $\left.{ }^{3} B_{1}, \mathrm{SiH}_{2}\right)$ and the MAD is $0.11^{\circ}$.

The SG-0 error $\left(4^{\circ}\right)$ for the dihedral angle in $\mathrm{HOOH}$ is disappointing, but the potential energy surface is very flat in this dimension and acute sensitivity to the quality of the quadrature should be expected.

\section{Harmonic Vibrational Frequencies}

Using the optimized geometries discussed above, 144 harmonic vibrational frequencies have been calculated at the same theoretical level. A selection of these frequencies and the corresponding zero-point energies are given in Table 4 . The full set is available as Supplementary Data. ${ }^{31}$ We note that, for molecules that possess nonabelian symmetry (e.g., a threefold rotation axis), the use of Lebedev grids (which possess octahedral symmetry) lifts the degeneracy of $E$ - and $T$-type vibrations. ${ }^{11}$ When this occurs, we have used the average value for comparisons. When the range of formally degenerate modes exceeds $1 \mathrm{~cm}^{-1}$, the difference is given in parentheses next to the average value.
Within the vibrational frequencies, for SG-1, the MAX is 76.9 $\mathrm{cm}^{-1}$ (lowest $A_{1}$ mode in $\mathrm{H}_{2} \mathrm{~S}$ ) and the MAD is $6.2 \mathrm{~cm}^{-1}$. For SG-0, the MAX is $80.9 \mathrm{~cm}^{-1}$ (the lowest $A_{1}$ mode in ${ }^{1} A_{1} \mathrm{SiH}_{2}$ ) and the MAD is $7.8 \mathrm{~cm}^{-1}$. Within the zero-point energies, for SG-1, the MAX is $343 \mu E_{h}$ (for $\mathrm{PH}_{2}$ ) and the MAD is $38 \mu E_{h}$. For SG-0, the MAX is $266 \mu E_{h}$ (for ${ }^{1} A_{l} \mathrm{SiH}_{2}$ ) and the MAD is $45 \mu E_{h}$.

\section{Summary}

The SG-0 grid appears capable of yielding a variety of physical quantities with tolerably small grid error. With the exception of bond angles, the SG-0 mean absolute deviation values are less than the twice the corresponding SG-1 values. Given that the SG-0 grid is only about half the size of the SG-1 grid, such increases in grid error are reasonable and support the use of SG-0 in production DFT calculations.

\section{Computation Time}

To assess the practical cost of the SG- 0 grid, we have compared B-LYP/6-31G(d) computation times when the SG-0 and SG-1 grids are used for a set of 20 amino acids. The molecular structures were taken from the database in the Spartan package and the calculations were performed on a 2-GHz dual-processor Power Macintosh under OSX version 10.4 operating system. 
Table 5 compares SG-1 and SG-0 on the basis of the total number of grid points, the cost of a geometry optimizations and the cost of a frequency calculation. On average, the SG-0 grid is almost exactly half the size of the SG-1 grid and this leads to a $50 \%$ speedup for the computations of the gradient and hessian of exchange-correlation energy, and a 35 and 38\% speedup in the total gradient and hessian computations, respectively.

\section{Conclusions}

By combining a sophisticated pruning technique with our MultiExp quadrature, we have constructed a new standard grid, SG-0, for DFT calculations. It consists of approximately 1500 grid points per first- or second-row atom, which is about half the size of the SG-1 grid, and generally yield grid errors that are roughly twice as large as those of SG-1. The reduction of grid-size results in a $50 \%$ CPU-time saving for gradient and hessian calculations for the exchange-correlation energy, and leads to a 35-38\% speedup in total gradient and hessian calculations. We believe that the SG-0 grid will be useful for DFT calculations on medium-to-large molecules and especially for preliminary structural studies. The SG-0 grid is the default quadrature for DFT calculations in the Q-Chem 3.0 package.

We thank the late Prof. John A. Pople for helpful suggestions, Dr. Jing Kong, Dr. Andrew Gilbert, and Q-Chem Inc. for technical support, and APAC for a generous grant of computer time. S.H.C. acknowledges $\mathrm{PhD}$ scholarships from the School of Chemistry (University of Nottingham) and the Research School of Chemistry (Australian National University).

\section{References}

1. Hohenberg, P.; Kohn, W., Phys Rev 1964, 136, B864.

2. Perdew, J. P.; Schmidt, K. In Density Functional Theory and Its Application to Materials; van Doren, V.; van Alsenoy C.; Geerlings, P., Eds.; AIP Conf. Proc. No. 577, Melville, NY, 2001.

3. Perdew, J. P.; Zunger, A. Phys Rev 1981, B 23, 5048.

4. Dirac, P. A. M. Proc Cam Philos Soc 1930 26, 376; Slater, J. C. Phys Rev 1951, 81, 385.

5. Zheng, Y. C.; Almlöf, J. Chem Phys Lett 1993, 214, 397; J Mol Struct (Theochem) 1996, 288, 277.

6. Werpetinski, K. S.; Cook, M. Phys Rev A 1995, 52, R3397; J Chem Phys 1997, 106, 7124.

7. Glaesemann, K. R.; Gordon, M. S. J Chem Phys 1998, 108, 9959; 1999, 110, 6580; 2000, 112, 10738.
8. Berghold, G.; Hutter, J.; Parrinello, M. Theor Chem Acc 1998, 99, 344.

9. Becke, A. D. J Chem Phys 1988, 88, 2547.

10. Murray, C. W.; Handy, N. C.; Laming, G. J. Mol Phys 1993, 78, 997.

11. Gill, P. M. W.; Johnson, B. G.; Pople, J. A. Chem Phys Lett 1993, 209, 506.

12. Treutler O.; Ahlrichs, R. J Chem Phys 1995, 102, 346.

13. Mura, M. E.; Knowles, P. J. J Chem Phys 1996, 104, 9848.

14. Lindh, R.; Malmqvist P. -A.; Gagliardi, L. Theor Chem Acc 2001, 106, 178.

15. Gill, P. M. W.; Chien, S. -H. J Comput Chem 2003, 24, 732.

16. El-Sherbiny, A.; Poirier, R. A. J Comput Chem 2004, 25, 1378.

17. Pérez-Jordá, J. M.; Becke A. D.; San-Fabián, E. J Chem Phys 1994, 100,6520 .

18. Köster, A. M.; Flores-Moreno, R.; Reveles, J. U. J Chem Phys 2004, $121,681$.

19. White, C. A.; Johnson, B. G.; Gill, P. M. W.; Head-Gordon, M. Chem Phys Lett 1994, 230, 8.

20. Fusti-Molnar, L.; Pulay, P. J Chem Phys 2002, 117, 7827; FustiMolnar, L.; Kong, J. J Chem Phys 2005, 122, 074108.

21. Kato, T. Commun Pur Appl Math 1957, 10, 151

22. Stroud, A. H. Approximate calculation of multiple integrals; PrenticeHall: Englewood Cliffs, NJ, 1971.

23. Lebedev, V. I. Zh Vychisl Mat Mat Fiz 1975, 15, 48; Zh Vychisl Mat Mat Fiz 1976, 16, 293; Sibirsk Mat Zh 1977, 18, 132.

24. Lebedev V. I.; Skorokhodov, A. L. Russian Acad Sci Dokl Math 1992, $45,587$.

25. Lebedev V. I.; Laikov, D. N. Dokl Akad Nauk 1999, 366, 741.

26. Koch, W.; Holthausen, M. C. A Chemists Guide to Density Functional Theory; Wiley-VCH: Weinheim, 2001.

27. Delley, B. J Comput Chem 1996, 17, 1152.

28. Kong, J.; White, C. A.; Krylov, A. I.; Sherrill, D.; Adamson, R. D.; Furlani, T. R.; Lee, M. S.; Lee, A. M.; Gwaltney, S. R.; Adams, T. R.; Ochsenfeld, C.; Gilbert, A. T. B.; Kedziora, G. S.; Rassolov, V. A.; Maurice, D. R.; Nair, N.; Shao, Y.; Besley, N. A.; Maslen, P. E.; Dombroski, J. P.; Daschel, H.; Zhang, W.; Korambath, P. P.; Baker, J.; Byrd, E. F. C.; Van Voorhis, T.; Oumi, M.; Hirata, S.; Hsu, C. P.; Ishikawa, N.; Florian, J.; Warshel, A.; Johnson, B. G.; Gill, P. M. W.; Head-Gordon, M.; Pople, J. A.; J Comput Chem 2000, 21, 1532.

29. Pople, J. A.; Head-Gordon, M.; Fox, D. J.; Raghavachari, K.; Curtiss, L. A. J Chem Phys 1989, 90, 5622; Curtiss, L. A.; Jones, C.; Trucks, G. W.; Raghavachari, K.; Pople, J. A. J Chem Phys 1990, 93, 2537 Curtiss, L. A.; Raghavachari, K.; Pople, J. A. J Chem Phys 1995, 103, 4192; Curtiss, L. A.; Raghavachari, K.; Trucks, G. W.; Pople, J. A. J Chem Phys 1992, 94, 7221.

30. Curtiss, L. A.; Raghavachari, K.; Redfern, P. C.; Rassolov, V.; Pople, J. A. J Chem Phys 1998, 109, 7764.

31. http://rsc.anu.edu.au/ pgill/research.php.

32. Martin, J. M. L.; Bauschlicher, C. W.; Ricca, A.; Comput Phys Commun 2001, 133, 189. 